\title{
Self-gravitating Envelope Solitons in a Degenerate Quantum Plasma System
}

\author{
N. Ahmed, N. A. Chowdhury, A. Mannan and A. A. Mamun \\ Department of Physics, Jahangirnagar University, Savar, Dhaka-1342, Bangladesh \\ Email: nahmed93phy@gmail.com
}

\begin{abstract}
The existence and the basic features of ion-acoustic (IA) envelope solitons in a selfgravitating degenerate quantum plasma system (SG-DQPS), containing inertial non-relativistically degenerate light and heavy ion species as well as inertialess non-relativistically degenerate positron and electron species, have been theoretically investigated by deriving the nonlinear Schrödinger (NLS) equation. The NLS equation, which governs the dynamics of the IA waves, has disclosed the modulationally stable and unstable regions for the IA waves. The unstable region allows to generate bright envelope solitons which are modulationaly stable. It is found that the stability and the growth rate are dependent on the plasma parameters (like, mass and number density of the plasma species). The implications of our results in astronomical compact object (viz. white dwarfs, neutron stars, and black holes, etc.) are briefly discussed.
\end{abstract}

Keywords: Modulational instability, reductive perturbation method, envelope solitons.

\section{Introduction}

The field of self-gravitating degenerate quantum plasma (DQP) physics is one of the current interesting research field among the plasma physics community because of the painstaking observational evidence which confirms the existence of such extreme plasma conditions in astronomical compact objects (viz. white dwarfs, neutron stars, and black holes, etc. [1-5]) and potential applications in modern technology (viz. metallic and semiconductor nano-structures, quantum x-ray free-electron lasers, nano-plasmonic devices $[6,7]$, metallic nano-particles, spintronics [8], thin metal films, nano-tubes, quantum dots, and quantum well [9], etc.). The number density of the plasma species is extremely high in self-gravitating DQP system (SG-DQPS) (order of $10^{30} \mathrm{~cm}^{-3}$ in white dwarfs $[2,10]$ and order of $10^{36} \mathrm{~cm}^{-3}$ or even more in neutron stars $[2,10]$ ) which leads to generate a strong gravitational field inside the plasma medium. Basically, the SG-DQPS contains degenerate inertial light (viz. ${ }_{1}^{1} \mathrm{H}[11,12]$ or ${ }_{2}^{4} \mathrm{He}[1,3]$ or ${ }_{6}^{12} \mathrm{C}[2,4]$ ) and heavy (viz. ${ }_{26}^{56} \mathrm{Fe}[13]$ or ${ }_{37}^{85} \mathrm{Rb}[14]$ or ${ }_{42}^{96} \mathrm{Mo}[14]$ ) ion species and inertialess degenerate electron and positron species. Heisenberg's uncertainty principle established the relationship between the uncertainty to determine the position and momentum of a particle simultaneously, and mathematically it can be expressed as, $\Delta x \Delta p \geq \hbar / 2$ (where $\Delta x$ is the uncertainty in position of the particle and $\Delta p$ is the uncertainty in momentum of the same particle, and $\hbar$ is the reduced Planck constant). This indicates that the position of the plasma species are very certain (because of highly dense and compressed plasma species) inside the plasma system but the momenta of the plasma species are extremely uncertain. Therefore these plasma species with uncertain in momentum give rise to a very high pressure known as "degenerate pressure". The expression for the degenerate pressure $P_{j}$ (degenerate plasma particle species $j$ ) as a function of number density $\left(N_{j}\right)$ is given by $[1,3,15]$

$$
P_{j}=K_{j} N_{j}^{\gamma}, \quad \gamma=\frac{5}{3}, \quad K_{j} \simeq \frac{3}{5} \frac{\pi \hbar^{2}}{m_{j}},
$$

where $j=e(p)$ for the electron (positron) species, and $j=l(h)$ for the light (heavy) ion species, respectively. The $\gamma$ is the relativistic factor $(\gamma=5 / 3$ stands for non-relativistic case and $\gamma=4 / 3$ stands for ultra-relativistic case) and $m_{j}$ is the mass. It is clear from (1) that the degenerate pressure $P_{j}$ is independent on thermal temperature but depends on degenerate particle number density $N_{j}$ and mass $m_{j}$. Finally, the strong gravitational field (degenerate pressure) of the SG-DQPS wants to squeeze 
(stretch) the plasma system but they are counter-balanced to each other. During the last few years, a large number of authors have studied the propagation of nonlinear waves in DQP by considering self-gravitational or without self-gravitational field. Asaduzzaman et al. [16] have investigated the linear and nonlinear propagation of self-gravitational perturbation mode in a SG-DQPS and found that selfgravitational perturbation mode becomes unstable when the wavelength of the perturbation mode is minimum. Mamun [17] examined the self-gravito shock structures in a SG-DQPS. Chowdhury et al. [18] have studied the modulational instability (MI) of nucleus-acoustic waves in a DQP system and found that the bright and dark envelope solitons are modulationally stable. But to the best of our knowledge, no attempt has been made to study MI of the ion-acoustic waves (IAWs) by deriving a nonlinear Schrödinger (NLS) equation and the formation of the envelope solitons in any kind of SG-DQPS. Therefore, in the present work, a SG-DQPS (containing inertialess degenerate electron and positron species, inertial degenerate light as well as heavy ion species) has been considered to obtain the conditions of MI of the IAWs and the formation of the envelope solitons, and also to identify their basic features.

The rest of the manuscript is organized as follows. The basic governing equations for the dynamics of the SG-DQPS are descried in Section 2. The derivation of the NLS equation is provided in Section 3. The stability of the IAWs and envelope solitons are examined in Section 4. A brief discussion is finally presented in Section 5.

\section{Governing Equations}

We consider a SG-DQPS containing inertialess degenerate electrons (mass $m_{e}$; number density $N_{e}$ ), positrons (mass $m_{p}$; number density $N_{p}$ ), inertial degenerate light ions (mass $m_{l}$; number density $N_{l}$ ), and heavy ions (mass $m_{h}$; number density $N_{h}$ ). The detail information about the light and heavy nuclei is provided in Table 1. The nonlinear dynamics of the SG-DQPS is described by

$$
\begin{aligned}
& \frac{\partial P_{e}}{\partial X}=-m_{e} N_{e} \frac{\partial \tilde{\phi}}{\partial X}, \\
& \frac{\partial P_{p}}{\partial X}=m_{p} N_{p} \frac{\partial \tilde{\phi}}{\partial X}, \\
& \frac{\partial N_{l}}{\partial T}+\frac{\partial}{\partial X}\left(N_{l} U_{l}\right)=0 \\
& \frac{\partial U_{l}}{\partial T}+U_{l} \frac{\partial U_{l}}{\partial X}=-\frac{\partial \tilde{\phi}}{\partial X}-\frac{1}{m_{l} N_{l}} \frac{\partial P_{l}}{\partial X}, \\
& \frac{\partial^{2} \tilde{\phi}}{\partial X^{2}}=4 \pi G\left(m_{l} N_{l}+m_{h} N_{h}+m_{e} N_{e}+m_{p} N_{p}\right),
\end{aligned}
$$

where $P_{e}, P_{p}$, and $P_{l}$ are the degenerate pressure of the degenerate electrons, positrons, and light ions, respectively; $X(T)$ is the space (time) variable; $U_{l}$ is the light ion fluid speed; $\tilde{\phi}$ is the self-gravitational potential; $G$ is the universal gravitational constant. We consider the SG-DQPS in which the charge densities of positive and negative plasma particle species fluctuate in such a way that the wave electric field always remains constant. Now, the charge neutrality condition for the electrostatic wave potential is

$$
N_{e}=N_{p}+Z_{l} N_{l}+Z_{h} N_{h}
$$

where $Z_{l}$ and $Z_{h}$ are the charge state of light and heavy ions, respectively. Here, it may be noted that the effect of the electrostatic wave potential has been neglected. Now, we consider normalized variables, namely, $x=X / L_{q}, t=T \omega_{j l}, n_{l}=N_{l} / n_{l 0}, u_{l}=U_{l} / C_{q}, C_{q}=\sqrt{\pi} \hbar n_{e 0}^{1 / 3} / m_{l}, \phi=\tilde{\phi} / C_{q}^{2}, \omega_{j l}^{-1}=\left(4 \pi G m_{l} n_{l 0}\right)^{-1 / 2}$ (where $n_{l 0}$ and $n_{e 0}$ are the equilibrium number densities of the light ion and electron species, respectively). 
After normalization, equations (2)-(6) can be written as

$$
\begin{aligned}
& \frac{\partial \phi}{\partial x}=-\frac{3}{2} \alpha^{2} \frac{\partial n_{e}^{2 / 3}}{\partial x}, \\
& \frac{\partial \phi}{\partial x}=\frac{3}{2} \sigma_{1}^{2} \sigma_{2}^{2 / 3} \frac{\partial n_{p}^{2 / 3}}{\partial x}, \\
& \frac{\partial n_{l}}{\partial t}+\frac{\partial}{\partial x}\left(n_{l} u_{l}\right)=0, \\
& \frac{\partial u_{l}}{\partial t}+u_{l} \frac{\partial u_{l}}{\partial x}=-\frac{\partial \phi}{\partial x}-\beta \frac{\partial n_{l}^{2 / 3}}{\partial x}, \\
& \frac{\partial^{2} \phi}{\partial x^{2}}=\gamma_{e}\left(n_{e}-1\right)-\gamma_{l}\left(n_{l}-1\right)+\gamma_{p}\left(n_{p}-1\right),
\end{aligned}
$$

where $\alpha=m_{l} / m_{e}, \sigma_{1}=m_{l} / m_{p}, \sigma_{2}=n_{p 0} / n_{e 0}, \mu=n_{e 0} / n_{l 0}, \beta=(3 / 2) \mu^{-2 / 3}, \lambda=n_{p 0} / n_{l 0}, \gamma=$ $Z_{l} m_{h} / Z_{h} m_{l}$ (which is greater than 1 for any set of heavy and light ion species), $\gamma_{e}=\mu\left(1 / \alpha+\gamma / Z_{l}\right)$ (here, $1 / \alpha \ll \gamma / Z_{l}$, where $1 / \alpha$ varies from $\sim 10^{-4}$ to $\sim 10^{-3}$, and $\gamma / Z_{l}$ varies from $\sim 0.1$ to 2.0 , and this means that $\left.\gamma_{e} \simeq \mu \gamma / Z_{l}\right), \gamma_{l}=\gamma-1, \gamma_{p}=\lambda\left(1 / \sigma_{1}-\gamma / Z_{l}\right)$. For inertialess degenerate electron and positron, the number densities can be expressed as

$$
\begin{aligned}
& n_{e}=\left(1-\frac{2 \phi}{3 \alpha^{2}}\right)^{\frac{3}{2}}, \\
& n_{p}=\left(1+\frac{2 \phi}{3 \sigma_{1}^{2} \sigma_{2}^{\frac{2}{3}}}\right)^{\frac{3}{2}} .
\end{aligned}
$$

Table 1. The values of $\gamma$ when ${ }_{1}^{1} \mathrm{H}[11,12],{ }_{2}^{4} \mathrm{He}[1]$, and ${ }_{6}^{12} \mathrm{C}[2,4]$ are considered as the light ion species, and ${ }_{26}^{56} \mathrm{Fe}[13],{ }_{37}^{85} \mathrm{Rb}[14]$, and ${ }_{42}^{96} \mathrm{Mo}[14]$ are considered as the heavy ion species.

\begin{tabular}{|c|c|c|}
\hline Light ion species & $\begin{array}{l}\text { Heavy } \\
\text { species }\end{array}$ & $\gamma$ \\
\hline \multirow{4}{*}{${ }_{1}^{1} \mathrm{H}[11,12]$} & ${ }_{26}^{56} \mathrm{Fe}[13]$ & 2.16 \\
\cline { 2 - 3 } & ${ }_{37}^{85} \mathrm{Rb}[14]$ & 2.30 \\
\cline { 2 - 3 } & ${ }_{42}^{96} \mathrm{Mo}[14]$ & 2.28 \\
\hline \multirow{5}{*}{${ }_{2}^{4} \mathrm{He}[1]$} & ${ }_{26}^{56} \mathrm{Fe}[13]$ & 1.08 \\
\cline { 2 - 3 } & ${ }_{37}^{85} \mathrm{Rb}[14]$ & 1.15 \\
\cline { 2 - 3 } & ${ }_{42}^{96} \mathrm{Mo}[14]$ & 1.14 \\
\hline \multirow{5}{*}{${ }_{6}^{12} \mathrm{C}[2,4]$} & ${ }_{26}^{56} \mathrm{Fe}[13]$ & 1.08 \\
\cline { 2 - 3 } & ${ }_{37}^{85} \mathrm{Rb}[14]$ & 1.15 \\
\cline { 2 - 3 } & ${ }_{42}^{96} \mathrm{Mo}[14]$ & 1.14 \\
\hline
\end{tabular}

Now, we substitute equations (13) and (14) into (12) and extend the resulting equation up to third order in $\phi$, we get

$$
\frac{\partial^{2} \phi}{\partial x^{2}}-\gamma_{l}+\gamma_{l} n_{l}=\gamma_{1} \phi+\gamma_{2} \phi^{2}+\gamma_{3} \phi^{3}+\ldots
$$


where

$$
\gamma_{1}=\left(\frac{\gamma_{p}}{\sigma_{1}^{2} \sigma_{2}^{2 / 3}}-\frac{\gamma_{e}}{\alpha^{2}}\right), \quad \gamma_{2}=\left(\frac{\gamma_{e}}{6 \alpha^{4}}+\frac{\gamma_{p}}{6 \sigma_{1}^{4} \sigma_{2}^{4 / 3}}\right), \quad \gamma_{3}=\left(\frac{\gamma_{e}}{54 \alpha^{6}}-\frac{\gamma_{p}}{54 \sigma_{1}^{6} \sigma_{2}^{2}}\right)
$$

We note that the terms on the right hand side of (15) are the contribution of electron and positron species. Thus, equations (10), (11), and (15) describe the dynamics of the gravitational envelop solitons in the SG-DQPS under consideration.

\section{Derivation of the NLS Equation}

To investigate the MI of the IA waves in SG-DQPS, we will derive the NLS equation by employing the reductive perturbation method $[19,20]$. So, we first introduce the stretched co-ordinates for independent variables $x$ and $t$ in terms of $\xi$ and $\tau$ as follows:

$$
\left.\begin{array}{l}
\xi=\epsilon\left(x-v_{g} t\right) \\
\tau=\epsilon^{2} t
\end{array}\right\}
$$

where $v_{g}$ is the envelope group velocity and $\epsilon$ is a small dimensionless expansion parameter. Then we can expand all dependent physical variables $n_{l}, u_{l}$, and $\phi$ in power series of $\epsilon$ as

$$
\begin{aligned}
& n_{l}=1+\sum_{m=1}^{\infty} \epsilon^{(m)} \sum_{l^{\prime}=-\infty}^{\infty} n_{l l^{\prime}}^{(m)}(\xi, \tau) \exp \left[i l^{\prime}(k x-w t)\right], \\
& u_{l}=\sum_{m=1}^{\infty} \epsilon^{(m)} \sum_{l^{\prime}=-\infty}^{\infty} u_{l l^{\prime}}^{(m)}(\xi, \tau) \exp \left[i l^{\prime}(k x-w t)\right] \\
& \phi=\sum_{m=1}^{\infty} \epsilon^{(m)} \sum_{l^{\prime}=-\infty}^{\infty} \phi_{l^{\prime}}^{(m)}(\xi, \tau) \exp \left[i l^{\prime}(k x-w t)\right]
\end{aligned}
$$

where $k(\omega)$ is the real variable representing the fundamental carrier wave number (frequency). The derivative operators in (10), (11), and (15) are regarded as

$$
\begin{aligned}
\frac{\partial}{\partial t} \rightarrow \frac{\partial}{\partial t}-\epsilon v_{g} \frac{\partial}{\partial \xi}+\epsilon^{2} \frac{\partial}{\partial \tau}, \\
\frac{\partial}{\partial x} \rightarrow \frac{\partial}{\partial x}+\epsilon \frac{\partial}{\partial \xi} .
\end{aligned}
$$

Now, by substituting (17)-(21) into (10), (11), and(15) and collecting the different powers of $\epsilon$. Now, the first order $(m=1)$ reduced equations with $l^{\prime}=1$ can be expressed as

$$
\begin{aligned}
& n_{l 1}^{(1)}=\frac{k^{2}}{S} \phi_{1}^{(1)}, \\
& u_{l 1}^{(1)}=\frac{k \omega}{S} \phi_{1}^{(1)},
\end{aligned}
$$

where $S=\omega^{2}-\beta_{1} k^{2}$ and $\beta_{1}=2 \beta / 3$. The compatibility condition of the system leads to the linear dispersion relation as

$$
\omega^{2}=\frac{\gamma_{l} k^{2}}{\gamma_{1}+k^{2}}+\beta_{1} k^{2}
$$

The dispersion characteristics of the wave are depicted in Fig. 1 [obtained from equation (24)], which indicates that (a) the angular wave frequency $(\omega)$ of the IAWs exponentially decreases with the increase 


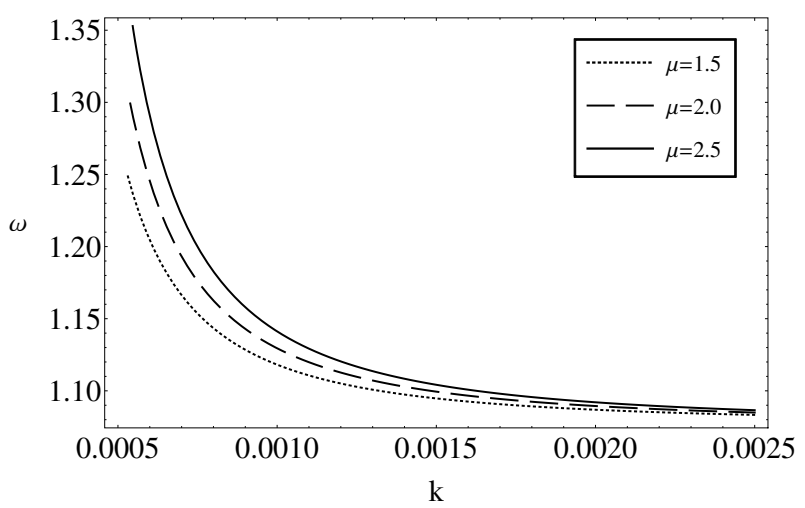

Figure 1. The variation of $\omega$ with $k$ for different values of $\mu$; along with $\alpha=3.67 \times 10^{3}, \gamma=2.16, \gamma / Z_{l}=0.5$, $\sigma_{1}=3.68 \times 10^{3}, \sigma_{2}=0.3$, and $\lambda=0.2$.

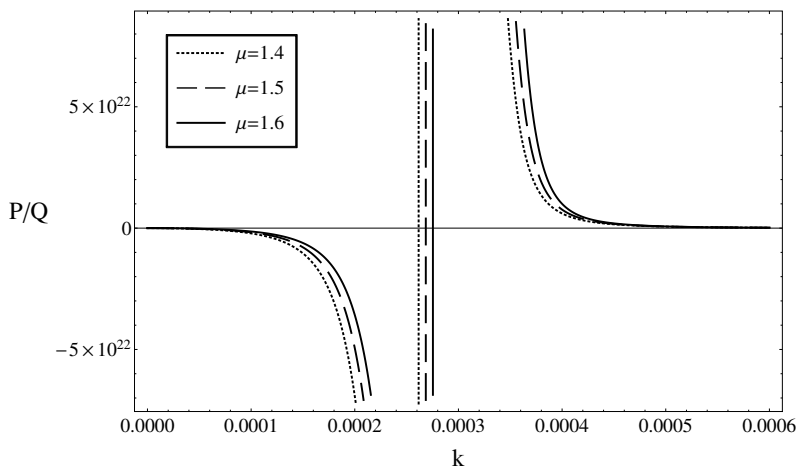

Figure 2. The variation of $P / Q$ with $k$ for different values of $\mu$; along with $\alpha=3.67 \times 10^{3}, \gamma=2.16, \gamma / Z_{l}=0.5$, $\sigma_{1}=3.68 \times 10^{3}, \sigma_{2}=0.3$, and $\lambda=0.2$.

of $k$; (b) the value of $\omega$ increases with the increase of $n_{e 0}$ for the fixed value of $n_{l 0}\left(\right.$ via $\left.\mu=n_{e 0} / n_{l 0}\right)$. The second order $(m=2)$ reduced equations with $l^{\prime}=1$ are given by,

$$
\begin{aligned}
& n_{l 1}^{(2)}=\frac{k^{2}}{S} \phi_{1}^{(2)}+\frac{2 i k \omega\left(k v_{g}-\omega\right)}{S^{2}} \frac{\partial \phi_{1}^{(1)}}{\partial \xi}, \\
& u_{l 1}^{(2)}=\frac{k \omega}{S} \phi_{1}^{(2)}+\frac{i\left(\omega^{2}+\beta_{1} k^{2}\right)\left(k v_{g}-\omega\right)}{S^{2}} \frac{\partial \phi_{1}^{(1)}}{\partial \xi},
\end{aligned}
$$

thus, the expression for $v_{g}$ is obtained as

$$
v_{g}=\frac{\partial \omega}{\partial k}=\frac{\gamma_{l} \omega^{2}-\left(\omega^{2}-\beta_{1} k^{2}\right)^{2}}{k \omega \gamma_{l}} .
$$

The amplitude of the second-order harmonics is found to be proportional to $\left|\phi_{1}^{(1)}\right|^{2}$

$$
\left.\begin{array}{l}
n_{l 2}^{(2)}=C_{1}\left|\phi_{1}^{(1)}\right|^{2}, \\
u_{l 2}^{(2)}=C_{2}\left|\phi_{1}^{(1)}\right|^{2}, \\
\phi_{2}^{(2)}=C_{3}\left|\phi_{1}^{(1)}\right|^{2}, \\
n_{l 0}^{(2)}=C_{4}\left|\phi_{1}^{(1)}\right|^{2}, \\
u_{l 0}^{(2)}=C_{5}\left|\phi_{1}^{(1)}\right|^{2}, \\
\phi_{0}^{(2)}=C_{6}\left|\phi_{1}^{(1)}\right|^{2},
\end{array}\right\}
$$


where the coefficients are

$$
\begin{aligned}
C_{1} & =\frac{2 C_{3} k^{2} S^{2}+3 \omega^{2} k^{4}}{2 S^{3}}, \\
C_{2} & =\frac{C_{1} \omega S^{2}-\omega k^{4}}{k S^{2}}, \\
C_{3} & =\frac{3 \gamma_{l} \omega^{2} k^{4}-2 \gamma_{2} S^{3}}{2 S^{3}\left(\gamma_{1}+4 k^{2}\right)-2 \gamma_{l} k^{2} S^{2}}, \\
C_{4}= & \frac{C_{6} S^{2}+k^{2} \omega^{2}+2 \omega v_{g} k^{3}-\beta_{2} k^{4}}{S^{2}\left(v_{g}^{2}-\beta_{1}\right)} \\
C_{5}= & \frac{C_{4} v_{g} S^{2}-2 \omega k^{3}}{S^{2}}, \quad \beta_{2}=\beta / 9, \\
C_{6}= & \frac{\left(k^{2} \omega^{2}+2 \omega v_{g} k^{3}-\beta_{2} k^{4}\right) \gamma_{l}-2 \gamma_{2} S^{2}\left(v_{g}^{2}-\beta_{1}\right)}{\gamma_{1} S^{2}\left(v_{g}^{2}-\beta_{1}\right)-\gamma_{l} S^{2}} .
\end{aligned}
$$

Finally, by substituting all the $(22)-(28)$ into the third order part $(m=3)$ and $l^{\prime}=1$ and simplifying them, we can obtain the following NLS equation:

$$
i \frac{\partial \Phi}{\partial \tau}+P \frac{\partial^{2} \Phi}{\partial \xi^{2}}+Q|\Phi|^{2} \Phi=0,
$$

where $\Phi=\phi_{1}^{(1)}$ for simplicity. The coefficient of dispersion and nonlinear terms $P$ and $Q$ are given by

$$
\begin{aligned}
& P=\frac{4 \beta_{1} k^{2} \omega^{3}+2 \beta_{1} v_{g} \omega^{2} k^{3}+v_{g} \beta_{1}^{2} k^{5}-4 \omega \beta_{1}^{2} k^{4}-3 k v_{g} \omega^{4}}{2 \gamma_{l} k^{2} \omega^{2}} \\
& Q=\frac{S^{2}\left[3 \gamma_{3}+2 \gamma_{2}\left(C_{3}+C_{6}\right)-F_{1}\right]}{2 \gamma_{l} \omega k^{2}}
\end{aligned}
$$

where $F_{1}=\left(k^{2} / S^{2}\right)\left[2 \omega k \gamma_{l}\left(C_{2}+C_{5}\right)+\gamma_{l} \omega^{2}\left(C_{1}+C_{4}\right)+\left(\gamma_{l} \beta_{3} k^{6} / S^{2}\right)\right]$, and $\beta_{3}=4 \beta / 81$.

\section{Stability Analysis and Envelope Solitons}

Let us now analyse the MI of IAWs by considering the linear solution of the NLS equation (29) in the form $\Phi=\hat{\Phi} e^{i Q|\hat{\Phi}|^{2} \tau}+c . c$ (c. c denotes the complex conjugate), where $\hat{\Phi}=\hat{\Phi}_{0}+\epsilon \hat{\Phi}_{1}$ and $\hat{\Phi}_{1}=\hat{\Phi}_{1,0} e^{i(\tilde{k} \xi-\tilde{\omega} \tau)}+$ c. c. Now, by substituting these values into (29), one readily obtains the following nonlinear dispersion relation $[18,21-25]$

$$
\tilde{\omega}^{2}=P^{2} \tilde{k}^{2}\left(\tilde{k}^{2}-\frac{2\left|\hat{\Phi}_{0}\right|^{2}}{P / Q}\right) .
$$

Here, the perturbed wave number $\tilde{k}$ and the perturbed frequency $\tilde{\omega}$ are different from the carrier wave number $k$ and frequency $\omega$. It is observed from (32) that the IAWs will be modulationally stable (unstable) in SG-DQPS for that range of values of $\tilde{k}$ in which $P / Q$ is negative (positive), i.e., $P / Q<0(P / Q>0)$. When $P / Q \rightarrow \pm \infty$, the corresponding value of $k\left(=k_{c}\right)$ is known as the critical or threshold wave number $\left(k_{c}\right)$ for the onset of MI. The variation of $P / Q$ with $k$ for $\mu$ is shown in Fig. 2 and which clearly indicates that (a) the IAWs are modulatonally stable (unstable) in SG-DQPS for small (long) wavelength; (b) the $k_{c}$ increases with the increase of $n_{e 0}$ for constant value of $n_{l 0}$ (via $\mu=n_{e 0} / n_{l 0}$ ). In the modulationally unstable $(P / Q>0)$ region and under this condition $\tilde{k}<\tilde{k}_{c}=\sqrt{2\left|\hat{\Phi}_{0}\right|^{2}(Q / P)}$, the MI growth rate can be written [from (32)] as

$$
\Gamma=|P| \tilde{k}^{2} \sqrt{\frac{\tilde{k}_{c}^{2}}{\tilde{k}^{2}}-1} .
$$




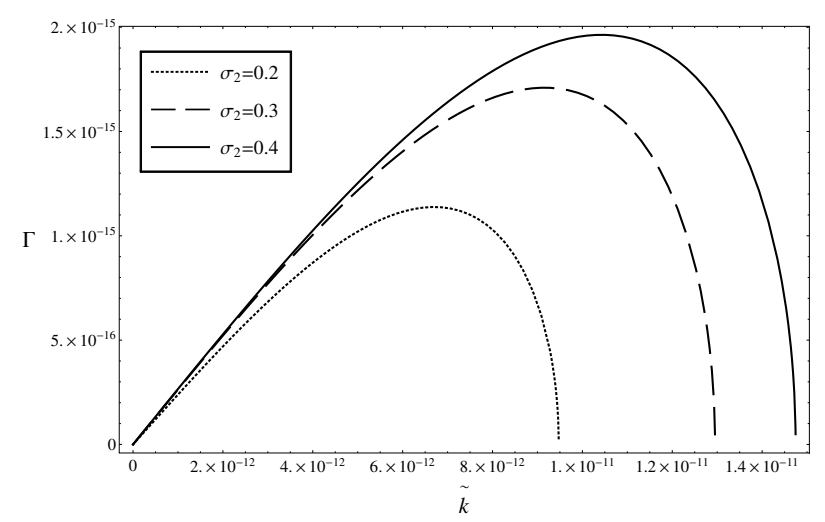

Figure 3. The variation of $\Gamma$ with $k$ for different values of $\sigma_{2}$; along with $\mu=1.5, \alpha=3.67 \times 10^{3}, \gamma=2.16$, $\gamma / Z_{l}=0.5, \sigma_{1}=3.68 \times 10^{3}, \lambda=0.2, k=0.0004$, and $\phi=0.8$.

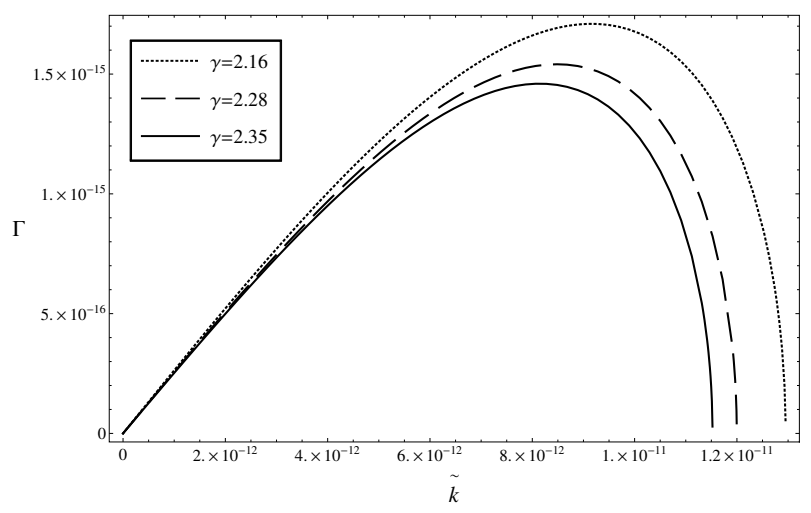

Figure 4. The variation of $\Gamma$ with $k$ for different values of $\gamma$; along with $\mu=1.5, \alpha=3.67 \times 10^{3}, \gamma / Z_{l}=0.5$, $\sigma_{1}=3.68 \times 10^{3}, \sigma_{2}=0.3, \lambda=0.2, k=0.0004$, and $\phi=0.8$.

The effect of $\sigma_{2}$ and $\gamma$ on the growth rate are presented in Figs. 3 and 4, where $\Gamma$ is plotted against $\tilde{k}$ and it is observed that (a) the growth rate $(\Gamma)$ increases with the increase in the value of positron number density $n_{p 0}$, but decreases with increase of the electron number density $n_{e 0}\left(\right.$ via $\left.\sigma_{2}=n_{p 0} / n_{e 0}\right)$; (b) the maximum value of $\Gamma$ increases (decreases) with the decrease of $m_{h}\left(m_{l}\right)$ for the fixed value of $Z_{l}$ and $Z_{h}$ (via $\gamma=Z_{l} m_{h} / Z_{h} m_{l}$ ); (c) on the other hand, the maximum value of $\Gamma$ increases (decreases) with the decrease of $Z_{l}\left(Z_{h}\right)$ for the fixed value of $m_{h}$ and $m_{l}$ (via $\left.\gamma=Z_{l} m_{h} / Z_{h} m_{l}\right)$. So, the charge state and mass of the light and heavy ion plays an opposite role to manifest the $\Gamma$ in SG-DQPS. The physics of this result is that the nonlinearity of the SG-DQPS increases (decreases) with the increase of the value of $m_{l}$ or $Z_{h}\left(m_{h}\right.$ or $\left.Z_{l}\right)$ which enhance (suppress) the maximum value of the $\Gamma$.

The self-gravitating bright envelop solitons are generated in the modulationally unstable region (when $P / Q>0)$ and the solitonic solution of (29) for the self-gravitating bright envelope solitons can be written as $[18,21-24]$

$$
\Phi(\xi, \tau)=\left[\psi_{0} \operatorname{sech}^{2}\left(\frac{\xi-U \tau}{W}\right)\right]^{1 / 2} \times \exp \left[\frac{i}{2 P}\left\{U \xi+\left(\Omega_{0}-\frac{U^{2}}{2}\right) \tau\right\}\right]
$$

where $U$ is the propagation speed of the localized pulse, $W$ is the pulse width which can be written as $W=\sqrt{2|P / Q| / \psi_{0}}\left(\psi_{0}\right.$ is the constant amplitude), and $\Omega_{0}$ is the oscillating frequency for $U=0$. The self-gravitating bright envelop solitons which are obtained from the numerical analysis of (34), are depicted 


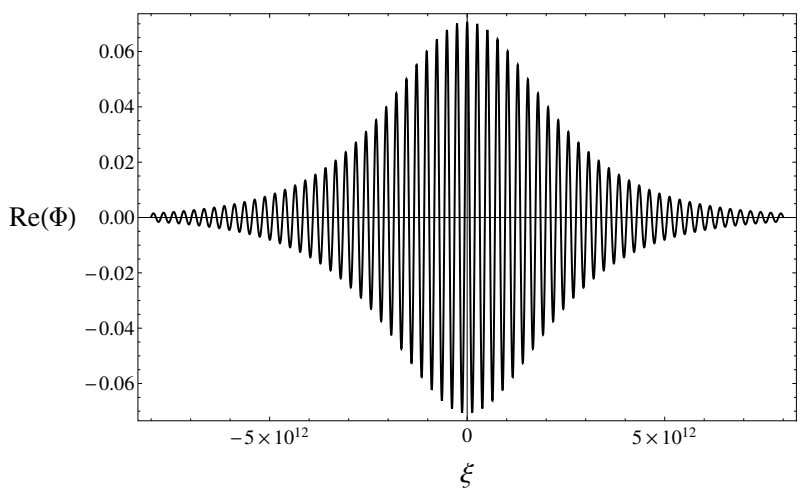

Figure 5. The variation of $\operatorname{Re}(\Phi)$ with $\xi$ for bright envelope solitons; along with $\mu=1.5, \alpha=3.67 \times 10^{3}, \gamma=2.16$, $\gamma / Z_{l}=0.5, \sigma_{1}=3.68 \times 10^{3}, \sigma_{2}=0.3, \lambda=0.2, U=0.001, k=0.0004, \psi_{0}=0.005, \tau=0, \Omega_{0}=0.04$.

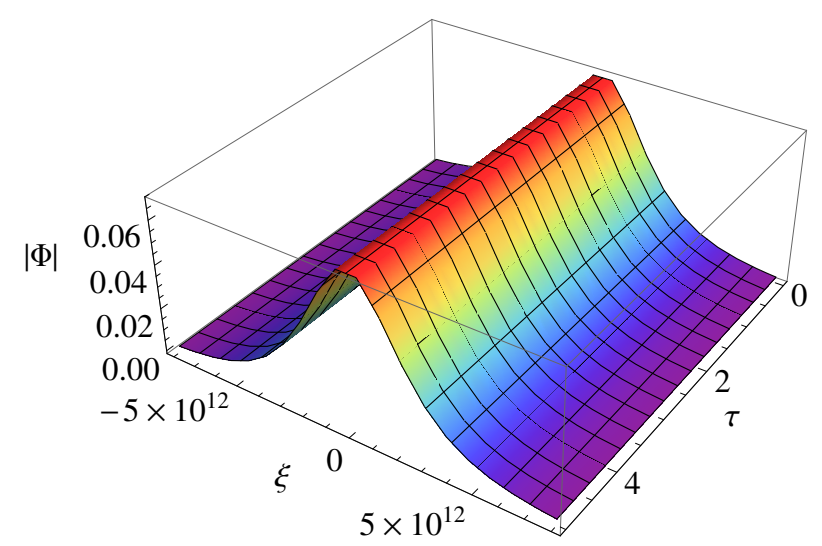

Figure 6. The variation of the $|\Phi|$ with $\xi$ and $\tau$ for bright envelope solitons; along with $\mu=1.5, \alpha=3.67 \times 10^{3}$, $\gamma=2.16, \gamma / Z_{l}=0.5, \sigma_{1}=3.68 \times 10^{3}, \sigma_{2}=0.3, \lambda=0.2, U=0.001, k=0.0004, \psi_{0}=0.005, \tau=0, \Omega_{0}=0.04$.

in Figs. 5 and 6 . The bright envelop solitons remain same as time $(\tau)$ passes, i.e., the self-gravitating bright envelop solitons are modulationally stable (please see Fig. 6).

\section{Discussion}

In our above analysis, we have considered an unmagnetized realistic laboratory or astrophysical SGDQPS consisting of inertialess non-relativistically degenerate electron and positron species, inertial non-relativistically degenerate light ion species as well as heavy ion species. The NLS equation has been derived by employing the well-known reductive perturbation method, which governs the evolution of nonlinear IAWs. The notable informations that have been found from our theoretical investigation, can be pin-pointed as follows:

1. The angular wave frequency $(\omega)$ of the IAWs exponentially decreases with the increase of $k$. On the other hand, the value of $\omega$ increases with the increase of $n_{e 0}$ for the fixed value of $n_{l 0}$ (via $\left.\mu=n_{e 0} / n_{l 0}\right)$.

2. The IAWs will be modulationally stable (unstable) for that range of values of $k$ in which $P / Q$ is negative (positive), i.e., $P / Q<0(P / Q>0)$.

3. The growth rate $(\Gamma)$ increases with the increase in the value of positron number density $n_{p 0}$, but decreases with increase of the electron number density $n_{e 0}\left(\right.$ via $\left.\sigma_{2}=n_{p 0} / n_{e 0}\right)$. On the other hand, the maximum value of $\Gamma$ increases (decreases) with the decrease of $m_{h}\left(m_{l}\right)$ for the fixed value of $Z_{l}$ 
and $Z_{h}$ (via $\gamma=Z_{l} m_{h} / Z_{h} m_{l}$ ). Furthermore, the maximum value of $\Gamma$ increases (decreases) with the decrease of $Z_{l}\left(Z_{h}\right)$ for the fixed value of $m_{h}$ and $m_{l}$ (via $\left.\gamma=Z_{l} m_{h} / Z_{h} m_{l}\right)$.

4. The self-gravitating bright envelop solitons remain same (modulationally stable) as time passes.

The findings of this theoretical investigation may be useful for understanding the nonlinear structure (bright envelope solitons) of a SG-DQPS in space (viz. neutron stars and white dwarf [1-5]).

\section{References}

1. S. Chandrasekhar, Astrophys. J. 7481 (1931).

2. D. Koester and G. Chanmugam, Rep. Prog. Phys. 53837 (1990).

3. R. H. Fowler, J. Astrophys. Astr. 15115 (1994).

4. D. Koester, Astron. Astrophys. Rev. 1133 (2002).

5. D. M. S. Zaman, M. Amina, P. R. Dip, and A. A. Mamun, Eur. Phys. J. Plus 132457 (2017).

6. H. A. Atwater, Sci. Am. 29656 (2007).

7. M. I. Stockmann, Phys. Today 6439 (2011).

8. S. A. Wolf, D. Awschalom, and R. A. Buhrman, Science 2941488 (2001).

9. G. Manfredi and P. A. Hervieux, Appl. Phys. Lett. 91061108 (2007).

10. S. L. Shapiro and S. A. Teukolsky, Black Holes, White Dwarfs and Neutron Stars: the Physics of Compact Objects (John Wiley \& Sons, New York, 1983).

11. R. S. Fletcher, X. L. Zhang, and S. L. Rolston, Phys. Rev. Lett. 96105003 (2006).

12. T. C. Killian, Nature (London) 441297 (2006).

13. A. Vanderburg et al., Nature (London) 526546 (2015).

14. A. Witze, Nature 510196 (2014).

15. A. A. Mamun and P. K. Shukla, Europhys. Lett. 9465002 (2011).

16. M. Asaduzzaman, A. Mannan, and A. A. Mamun, Phys. Plasmas 24052102 (2017).

17. A. A. Mamun, Phys. Plasmas 24102306 (2017).

18. N. A. Chowdhury, M. M. Hasan, A. Mannan, and A. A. Mamun, Vacuum 14731 (2018).

19. T. Taniuti and N. Yajima, J. Math. Phys. 101369 (1969).

20. N. A. Chowdhury, A. Mannan, M. M. Hasan, and A. A. Mamun, Chaos 27093105 (2017).

21. S. Sultana and I. Kourakis, Plasma Phys. Control. Fusion 53045003 (2011).

22. R. Fedele and H. Schamel, Eur. Phys. J. B 27313 (2002).

23. I. Kourakis and P. K. Shukla, Nonlinear Proc. Geophys. 12407 (2005).

24. R. Fedele and H. Schamel, Eur. Phys. J. B 27313 (2002).

25. N. A. Chowdhury, A. Mannan, and A. A. Mamun, Phys. Plasmas 24113701 (2017). 